\title{
Magnetization switching in bistable nanomagnets by picosecond pulses of surface acoustic waves
}

\author{
Vladimir S. Vlasov ${ }^{1,2},{ }^{*}$ Alexey M. Lomonosov ${ }^{1,3,4},{ }^{\dagger}$ Anton V. Golov ${ }^{2}$, Leonid N. Kotov ${ }^{2}$, Valentin \\ Besse $^{1}$, Alexandr Alekhin ${ }^{1}$, Dmitry A. Kuzmin ${ }^{5,6}$, Igor V. Bychkov ${ }^{5,6}$, and Vasily V. Temnov ${ }^{1 \ddagger}$ \\ ${ }^{1}$ Institut des Molécules et Matériaux du Mans, UMR CNRS 6283, Le Mans Université, 72085 Le Mans, France \\ ${ }^{2}$ Syktyvkar State University named after Pitirim Sorokin, 167001, Syktyvkar, Russia \\ ${ }^{3}$ Prokhorov General Physics Institute of the Russian Academy of Sciences, 119991, Moscow, Russia \\ ${ }^{4}$ Scientific-Technical Center for Unique Instrument Engineering, RAS, Moscow, Russia \\ ${ }^{5}$ Chelyabinsk State University, 454001 Chelyabinsk, Russia and \\ ${ }^{6}$ South Ural State University (National Research University), 454080 Chelyabinsk, Russia
}

(Dated: November 11, 2020)

\begin{abstract}
We perform a theoretical investigation of the magnetization switching in polycrystalline $\mathrm{Ni}$ nanoparticles induced by ultrashort pulses of surface acoustic waves via magneto-elastic interactions. In our numerical simulations, a Ni nanoparticle is modelled as an ellipsoidal disc deposited on a dielectric substrate. The in-plane external magnetic field breaks the symmetry and allows to adjust the height of the energy barrier between two metastable magnetization states of the free energy density and dramatically lower the amplitude of elastic strain pulses required for magnetization switching. The switching threshold is shown to depend on the duration of an acoustic pulse, the magnetic shape anisotropy of an elliptical nanoparticle, the amplitude of the external magnetic field, and the magneto-striction coefficient. We introduce the magneto-elastic switching diagram allowing for the simultaneous visualization of the switching threshold and its characteristic time-scale as a function of various physical parameters.
\end{abstract}

PACS numbers: Valid PACS appear here

\section{INTRODUCTION}

The interaction of bulk acoustic phonons and surface acoustic waves (SAWs) with magnetization in thin ferromagnetic films attracted a significant interest motivated by beautiful physics and new principles of magnetic recording [1-16].

Magnetization switching using quasi-monochromatic SAW pulses has a long history. Nonresonant magnetoelastic switching in micrometer-sized cobalt bars using $\mathrm{MHz}$-frequency SAWs has been demonstrated by Davis at al. [17]. Under similar excitation conditions, Sampath et al. reported on a novel magneto-acoustic mechanism for switching from a single-domain to vortex states using arrays of cobalt nanoellipses [18]. Experiments on a dilute magnetic semiconductor $(\mathrm{Ga}, \mathrm{Mn})(\mathrm{As}, \mathrm{P})$ evidenced both the non-resonant [19] and resonant [20] precessional magnetization switching. Assuming that time-dependent "ramped" voltages with picosecond rise times are applied to a piezoelectric transducer (PZT), Roy et al. have theoretically identified the regimes of ultralow loss $(\sim 100 \mathrm{kT})$ energy dissipation and sub-nanosecond switching times in ferromagnet/PZT multiferroic nanomagnets [21].

Here we explore an alternative route of magneto-elastic switching using single broadband SAW pulses of picosecond duration. In our previous work, we have predicted the phenomenon of the acoustically induced magnetization switching in Terfenol thin films using ultrafast acoustic strain pulses characterized by a relatively large strain

\footnotetext{
*vlasovvs@syktsu.ru

$\dagger$ lom@kapella.gpi.ru

‡ vasily.temnov@univ-lemans.fr
}

amplitude $\sim 1 \%$ [22]. Although acoustic pulses with such huge amplitude can be generated under strong excitation conditions [23], the energy barrier between the meta-stable states in Terfenol (determined by the competition of magneto-elastic, magnetocrystalline and magnetic shape anisotropies) can not be easily tuned.

On the other hand, recent all-optical transient grating (TG) experiments provide a detailed picture of magnetoelastic interactions as monitored by the small-angle ferromagnetic resonance (FMR) precession induced by quasimonochromatic surface acoustic transients $[9,10]$. In these experiments, the estimated strain amplitudes did not exceed $0.1 \%$. SAWs with amplitudes up to $0.3 \%$ have been measured recently in femtosecond TG experiments by ultrafast X-ray probing [24, 25]. A question arises if it is possible to design a magnetic system where the magnetization could be switched by readily available ultrashort surface acoustic transients with magnitudes not exceeding $0.1 \%$. Note that SAW amplitudes in femtosecond TG experiments appear to be significantly larger as compared to the typical SAW strains of the order of $10^{-5}$ generated by conventional interdigital transducers $[16,26]$.

Here we propose an in-plane architecture of a bistable magnetic system, where the magnetization can be switched by relatively weak ultrashort pulses of surface acoustic transients under the presence of a small external magnetic field. Interestingly, a similar generic system consisting of an elliptical nanoparticle driven by an oscillating magnetic field (under the static magnetic bias) has been investigated recently with the main focus on interplay between magnetization switching, quasiperiodicity and chaos [27, 28]. An ultralow energy threshold for single bit magnetization recording down to the ultimate 
Landauer limit [29] stimulated some recent experimental studies in ensembles of nanomagnets [30], isolated nanomagnets [31] and single atoms [32].

In this paper we focus on exploring the dynamics of acoustic magnetization switching induced by picosecond surface acoustic transients. In order to quantify the the switching behaviour in the multi-parameter space we introduce the magneto-elastic switching diagram visualizing the switching thresholds together with the characteristic switching time scale. From the technological point of view, the localized opto-acoustic excitation of picosecond SAW strain pulses using ultrashort laser pulses would allow for the contact-free magnetic recording while scanning the sample relative to the laser focus. Apart from that, this work represents the first step towards the understanding of the magnetization dynamics in a complex physical system: an anisotropic single-domain nanomagnet driven by a single-cycle SAW pulse with the characteristic time duration comparable to the natural frequency of FMR precession.

\section{GEOMETRY OF THE PROBLEM AND BASIC EQUATIONS}

We study a polycrystalline Ni nanoparticle in a shape of an ellipsoidal disc with a long axis $a$, a short axis $b$ and a thickness $c$. Such structures can be routinely fabricated from thin nickel films by electron or ion beam lithography. It is important to notice that such nanoparticles, produced by lithography techniques, stick to the surface and their mechanical adhesion is the same as that of the continuous thin film. Introducing the Cartesian coordinates, we can set the $y$ axis to be parallel to the long axis of the ellipse. The particle is placed in a permanent magnetic field $H$ which points in the positive direction of the $x$ axis. Short acoustic transient propagates along the $x$ axis, its front spreads along the $y$ axis.

The free energy density consists of Zeeman, demagnetization and magneto-elastic terms:

$$
F=F_{Z}+F_{d}+F_{m e}(t) .
$$

The Zeeman term reads:

$$
F_{Z}=-\mu_{0} M_{0} \mathbf{m} \cdot \mathbf{H}
$$

where $\mu_{0}$ is the magnetic permeability of vacuum, $M_{0}$ is the saturation magnetization of $\mathrm{Ni}, \mathbf{m}$ is the unit magnetization vector. The demagnetization energy

$$
F_{d}=\frac{1}{2} \mu_{0} M_{0}^{2} \mathbf{m} \cdot \mathbf{N} \cdot \mathbf{m}
$$

depends on the demagnetization tensor $\mathbf{N}$, and the magnetoelastic energy induced by a SAW propagating along the $\mathrm{x}$-axis

$$
F_{m e}(t)=b_{1} m_{x}^{2} e_{x x}(t)
$$

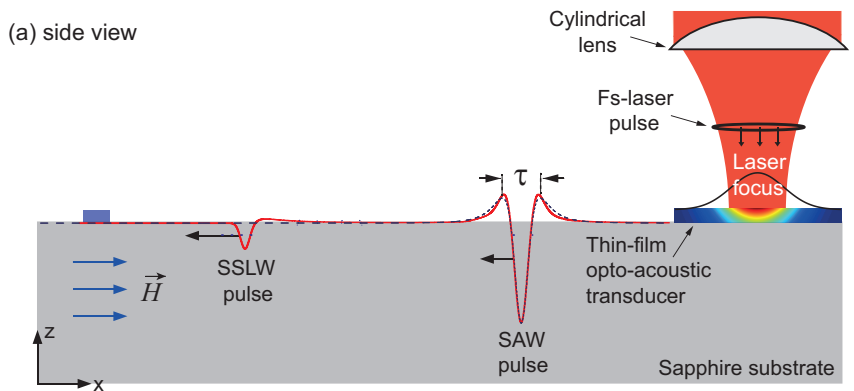

(b) top view

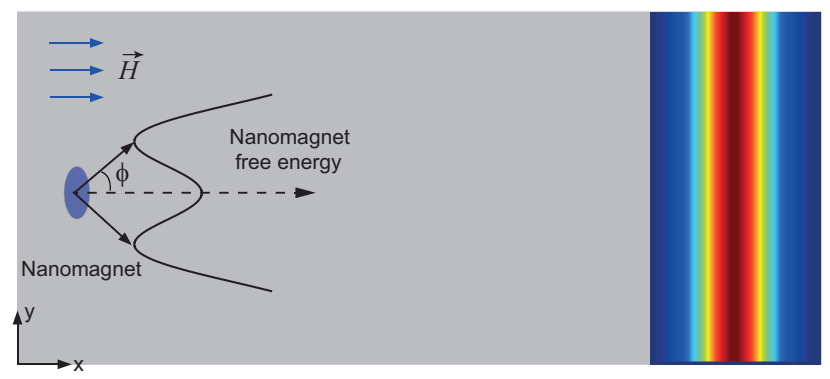

FIG. 1. Geometry of the problem: absorption of an ultrashort laser pulse in a thin-film opto-acoustic transducer launches picosecond surface acoustic transients (SSLW and SAW pulses, see Section 3 for details), which propagate along the surface and interact with a single elliptical nanomagnet via magnetostriction mechanism. A constant in-plane magnetic field is used to modify the free energy of the nanomagnet.

depends on the magnetoelastic constant $b_{1}=10^{7}\left[\mathrm{~J} / \mathrm{m}^{3}\right]$ (for nickel) and the time-dependent elastic strain $e_{x x}(t)$. Contribution of the other strain component $e_{z z}(t)$ can be neglected in the case $e_{z z} m_{z}<<e_{x x} m_{x}$, which is fulfilled in our simulations.

The demagnetization tensor $\mathbf{N}$ of an elliptical ferromagnetic nanoparticle has a following form:

$$
\mathbf{N}=\left(\begin{array}{ccc}
N_{x} & 0 & 0 \\
0 & N_{y} & 0 \\
0 & 0 & N_{z}
\end{array}\right)
$$

where the demagnetizing factors $N_{i}$ are determined by the particle dimensions $a, b, c$ via elliptic integrals of the first and second kind [33]. In order to calculate the elastically driven magnetization dynamics of the nickel nanoparticle we solve the Landau-Lifschitz-Gilbert (LLG) equation [34]

$$
\frac{\partial \mathbf{m}}{\partial t}=-\gamma \mu_{0} \mathbf{m}(t) \times \mathbf{H}_{e f f}(t)+\alpha \mathbf{m}(t) \times \frac{\partial \mathbf{m}}{\partial t}
$$

where $\gamma$ is the gyromagnetic ratio and $\alpha$ is the dimensionless phenomenological Gilbert damping. Projections of the effective time-dependent magnetic field $\mathbf{H}_{\text {eff }}(t)=$ $-\frac{1}{\mu_{0} M_{0}} \frac{\partial F}{\partial \mathbf{m}}$ on different axes read:

$$
\begin{aligned}
& H_{e f f, x}=-\left(N_{x} M_{0}+\frac{2 b_{1} e_{x x}}{\mu_{0} M_{0}}\right) m_{x}+H, \\
& H_{e f f, y}=-N_{y} M_{0} m_{y} \\
& H_{e f f, z}=-N_{z} M_{0} m_{z} .
\end{aligned}
$$


The vector equation (6) can be rewritten as the nonlinear system of equations for three components of the magnetization:

$$
\begin{array}{r}
\frac{d m_{x}}{d t}=-\frac{\gamma \mu_{0}}{1+\alpha^{2}}\left[\left(m_{y}+\alpha m_{x} m_{z}\right) H_{e f f, z}-\right. \\
\left.-\left(m_{z}-\alpha m_{y} m_{x}\right) H_{e f f, y}-\alpha\left(m_{y}^{2}+m_{z}^{2}\right) H_{e f f, x}\right] \\
\frac{d m_{y}}{d t}=-\frac{\gamma \mu_{0}}{1+\alpha^{2}}\left[\left(m_{z}+\alpha m_{y} m_{x}\right) H_{e f f, x}-\right. \\
\left.-\left(m_{x}-\alpha m_{z} m_{y}\right) H_{e f f, z}-\alpha\left(m_{z}^{2}+m_{x}^{2}\right) H_{e f f, y}\right] \\
\frac{d m_{z}}{d t}=-\frac{\gamma \mu_{0}}{1+\alpha^{2}}\left[\left(m_{x}+\alpha m_{z} m_{y}\right) H_{e f f, y}-\right. \\
\left.-\left(m_{y}-\alpha m_{x} m_{z}\right) H_{e f f, x}-\alpha\left(m_{x}^{2}+m_{y}^{2}\right) H_{e f f, z}\right] .
\end{array}
$$

We can tune free energy density by different means. For example, its demagnetization term $F_{d}$ depends on $N_{i}$, therefore it can be modified by varying the aspect ratio $a / b$ of the nanoparticle. In the presented analysis, we use the following particle dimensions: $a=150 \mathrm{~nm}$, $b=100 \mathrm{~nm}, c=20 \mathrm{~nm}$. The choice of these dimensions is arbitrary, but it fulfills certain requirements. First, such dimensions ensure that the magnetization is in a singledomain state and provide the volume of the nanoparticle to obtain the energy barrier between the metastable states in Fig. 2. Second, the small axis of the ellipse remains much smaller than the spatial extent of the SAW pulse and justifies the assumption of spatially homogeneous strain acting on the nanoparticle. Third, as long as the lateral dimensions of the nanoparticle are large compared to its thickness, i.e. $a, b \gg c$, the simulations will still be valid for nanodiscs with the same aspect ratio $a / b=1.5$. The macrospin approximation used throughout this manuscript should hold for characteristic dimensions between 10-300 nm [35].

Variation of the external magnetic field provides another flexible tool to change the structure of the free energy density. In the absence of the external magnetic field or for small $H$, the free energy $F$ possesses two minima corresponding to two meta-stable states of defined magnetization direction. Upon an increase of $H$, these minima merge into a single minimum, corresponding to the alignment of the magnetization along the external magnetic field.

To demonstrate this, Fig. 2 shows the cross-sections of the free energy density in the $x y$ plane for several values of the external magnetic field (Fig. 2(a)) and the dependence of the small-angle FMR precession on the external magnetic field (Fig. 2(b)).

Varying the amplitude $H$ of the static magnetic field, we can achieve two distinct situations: either two energy minima at $\pm \phi_{e q} \neq 0$ obeying

$$
\cos \phi_{e q}=\frac{H}{M_{0}\left(N_{x}-N_{y}\right)},
$$

for $H<H_{c r}$, or a single energy minimum at $\phi_{e q}=0$ for $H>H_{c r}$. The critical value of the magnetic field (in
Tesla), for which two energy minima merge into a single one reads:

$$
B_{c r}=\mu_{0} H_{c r}=\mu_{0} M_{0}\left(N_{x}-N_{y}\right) .
$$

For a given demagnetizing tensor with $N_{x}=$ $0.1385, N_{y}=0.0781, N_{z}=0.7835$ and $\mu_{0} M_{0}=0.6 \mathrm{~T}$ for nickel, critical magnetic field $B_{c r} \approx 37 \mathrm{mT}$ is reasonably low to be achieved by a conventional electromagnet. Variation of the external magnetic field can be used to tune continuously the height of the free energy barrier between two meta-stable magnetization states, which represents a practical advantage of the proposed geometry.

A strong variation of the FMR frequency in Fig. 2 (b) as a function of the external magnetic field, including its zero value at $B=B_{c r}$, can be associated with the change of curvature of the angular dependence of the free energy in the vicinity of $\phi=0$ from concave to convex. An analytical expression for the FMR frequency displayed in Fig. 2(b) reads:

$$
\begin{gathered}
f=\frac{\gamma \mu_{0}}{2 \pi}\left[M_{0}^{2}\left(N_{x}-N_{y}\right)\left(N_{z}-N_{y}\right) \sin ^{2} \phi-\left(M _ { 0 } \left(N_{z}-\right.\right.\right. \\
\left.\left.\left.-N_{x}\right) \cos \phi+H\right)\left(M_{0}\left(N_{x}-N_{y}\right) \cos \phi-H\right)\right]^{1 / 2} .
\end{gathered}
$$

This equation has been obtained by the linearization of the LLG equations in the vicinity of the equilibrium magnetization position $\phi_{e q}$. Analysis of the asymptotic behaviour of the equation (15) makes it easier to understand how the transition between these two characteristic cases occurs. At zero magnetic field $H=0$, the equilibrium magnetization is aligned along the long axis of the ellipse $\left(\phi_{e q}= \pm \pi / 2\right)$, i.e. the $Y$ axis, and the FMR frequency depends solely on the demagnetizing factors $N_{i}$ and the saturation magnetization $M_{0}$ :

$$
f=\frac{\gamma \mu_{0}}{2 \pi} M_{0}\left[\left(N_{x}-N_{y}\right)\left(N_{z}-N_{y}\right)\right]^{1 / 2} .
$$

For $H>H_{c r}$ the magnetization precession around the single energy minimum $\phi_{e q}=0$ occurs at an angular frequency:

$$
f=\frac{\gamma \mu_{0}}{2 \pi}\left[\left(M_{0}\left(N_{z}-N_{x}\right)+H\right)\left(H-M_{0}\left(N_{x}-N_{y}\right)\right)\right]^{1 / 2} .
$$

Dynamic perturbations of the bi-stable nanomagnet described above may result in magnetization switching. In this study we are particularly interested in the influence of transient elastic deformations induced by picosecond SAW pulses. In order to understand the behavior of the magnetization vector under the influence of the dynamic strain, it is instructive to show how the angular dependence of the free energy changes with a static strain, see Fig. 2(c). Whereas tension (positive strain $\epsilon_{x x}=5 \times 10^{-4}$ ) tends to shift the energy minima farther apart to larger angles and increase the height of the potential barrier between them, compression (negative strain $\epsilon_{x x}=-5 \times 10^{-4}$ ) makes the potential barrier disappear. It is worth mentioning that for a given value of the magnetic field $B=30 \mathrm{mT}$ the height of the 

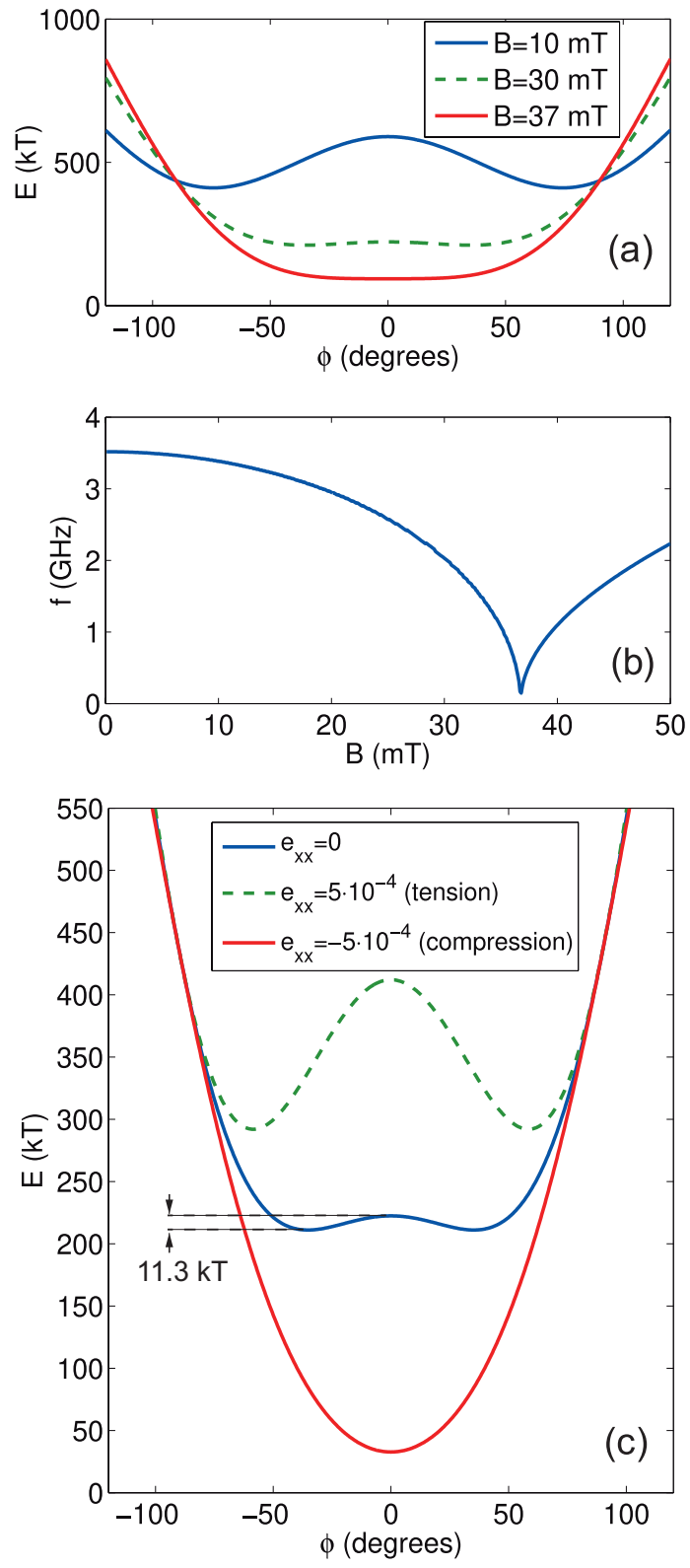

FIG. 2. (a) Dependence of the free energy on the azimuthal angle $\phi$, calculated for different values of the external magnetic field. (b) Dependence of a small-angle FMR precession on the magnetic field. (c) Influence of the static strain $e_{x x}=$ $\pm 5 \times 10^{-4}$ on the nanomagnet's free energy at $B=30 \mathrm{mT}$.

potential barrier 11.3 $k T(k T=25 \mathrm{meV}$ stands for thermal energy at room temperature) is very small. Therefore, energies required for magnetization switching are expected to approach the fundamental Landauer limit of $k T \ln 2=17 \mathrm{meV}$, i.e. the minimum energy required to record a single bit of information at $\mathrm{T}=300 \mathrm{~K}$ [29]. Having said that, it is not surprising that a relatively weak static strain with an amplitude of $\epsilon_{x x}= \pm 5 \times 10^{-4}$ can drastically modify the free energy density. The rest of the paper will address the following question: under which conditions a single ultrashort, picosecond SAW pulse can induce the magnetization switching, i.e. the transition from one metastable energy minimum to another one.

\section{GENERATION OF ULTRASHORT PULSES OF SURFACE ACOUSTIC WAVES}

Short acoustic pulses propagating at the surface of a solid can be generated by absorption of short laser pulses in a thin subsurface layer. This results in a transient and spatially inhomogeneous heating profile and subsequent generation of thermoelastic stress. This excites various acoustic modes in a solid, two of them propagate along the surface: surface skimming longitudinal wave (SSLW) and Rayleigh SAW [10,11].

By focusing a laser beam into a narrow strip one is able to generate an acoustic source with a one-dimensional spatial Gaussian distribution. Calculation of the acoustic response of sapphire surface (amorphous sapphire glass with Young's modulus of $345 \mathrm{GPa}$, Poisson's ratio 0.27 and mass density $3.98 \mathrm{~g} / \mathrm{cm}^{3}$ ) to such an excitation can be done as a convolution of the Green's function [36] with the source in the form of the Gaussian spatial stress distribution. The result exhibits two distinct pulses, the aforementioned SSLW and the SAW propagating with the longitudinal velocity $(10.4 \mu \mathrm{m} / \mathrm{ns})$ and Rayleigh velocity $(5.4 \mu \mathrm{m} / \mathrm{ns})$, respectively. Fig. 1 shows the strain component $e_{x x}$ associated with those waves. Note that negative or positive sign of $e_{x x}$ correspond to the material compression or stretching, respectively. Amplitude of the SSLW decays upon propagation due to a leakage into the bulk acoustic modes, whereas the SAW amplitude remains constant. Thus the nanoparticle positioned at a sufficiently large distance from the source will be affected solely by the SAW pulse. The dominant $e_{x x}(t)$ strain component of the SAW pulse generated in a thermoelastic regime by a thin Gaussian line source can be approximated by the following expression:

$$
e_{x x}(t)=\eta \cdot\left[\frac{4 p^{2}}{\left(1+p^{2}\right)^{3}}-\frac{1}{\left(1+p^{2}\right)^{2}}\right]
$$

where $p=2\left(t-t_{0}\right) / \tau, t_{0}$ is the position of the pulse center, $\tau$ and $\eta$ are the duration and the amplitude of the pulse, respectively. Here $\tau$ equals to the time interval between the two side maxima. The SAW $e_{x x}(t)$ pulse consists of a strong negative (compressional) peak surrounded by two relatively small positive symmetrical sidebands. The time integral of this model shape equals to zero, assuring the zero net displacement of the material after the SAW pulse has gone. This model pulse shape is shown in Fig. 1 together with the exact solution obtained through the Green's function method.

The model expression in the form of Eq. (18) will be used throughout of the manuscript to calculate the acoustically induced magnetization dynamics and diagrams of magnetization switching. The one-dimensional Gaussian focusing to $1 \mu \mathrm{m}$ generates a SAW pulse in sapphire substrate with the duration $\tau=280 \mathrm{ps}$, a value used in all calculations. 
The maximum possible amplitude $\eta$ of SAW pulses generated by the thermal expansion mechanism is ultimately limited by the damage threshold, which can be estimated by laser-induced heating of nickel close to its melting point $(1700 \mathrm{~K})$. Starting at room temperature, the corresponding temperature rise $\Delta T=1400 \mathrm{~K}$, multiplied by the thermal expansion coefficient $\beta=$ $13 \times 10^{-6} \mathrm{~K}^{-1}$ in $\mathrm{Ni}$, provides a rough estimate for the peak thermoelastic strain $\sim \beta \Delta T=1.8 \times 10^{-2}$. A more accurate estimate takes into account the Gaussian temperature distribution across the laser spot generating the spatially inhomogeneous thermoelastic stress $\sigma=\left(C_{11}+2 C_{12}\right) \beta \Delta T=10 \mathrm{GPa}$ acting on the surface of the sapphire substrate. Convolution of the Green's function [9] with such a Gaussian source provides the estimate for the maximum possible SAW amplitude $\eta \simeq 10^{-2}$. The simulations of magneto-elastic switching are performed for much smaller SAW amplitudes assuring that the fs-laser excitation fluence stays well below the damage threshold.

It is worth mentioning that ultrashort single-cycle acoustic SAW pulses at absorbing surfaces can be generated by tightly focused fs-laser pulses [37, 38], with SAW duration determined by the size of the focal spot divided by SAW-velocity. Using an elastically hard substrate such as sapphire should allow for reducing the SAWduration down to a few hundreds of picoseconds while focusing pump pulses of visible light through a high-NA microscope objective.

\section{SWITCHING DYNAMICS AND SWITCHING-TIME DIAGRAMS}

Let us inspect the magnetization dynamics following the excitation by an ultrashort acoustic pulse of the shape given by Eq. (18). We evaluate these dynamics by solving the LLG equations (10-12) numerically with the 4 th or 5th-order Runge-Kutta method.

Initially the magnetization vector rests in one of the two metastable minima (we choose a positive azimuthal angle). Impact of the acoustic pulse pushes the magnetization vector $\mathbf{m}$ via the magnetoelastic interaction given by Eq. (4), and its further motion is governed by the LLG equations. Depending on the magnitude of the strain pulse, the magnetization vector may either return to its initial steady position (Fig. 3(a) and (c)) or switch to another free energy minimum (Fig. 3(b)).

In addition to the amplitude of SAW pulses, which can be controlled by changing the intensity of optical pump pulses, the value of the external magnetic field can determine whether the magnetization will switch or not, see Fig. 4. Besides the fact that the magnetic field controls the switching in a similar manner as the amplitude of SAW pulse in Fig. 3, another remarkable observation concerns the characteristic time of the magnetization dynamics. At small values of the magnetic field $(20 \mathrm{mT}$ and $27 \mathrm{mT}$ in Fig. 4(a,b)) the time scale is determined by the period of the small-angle FMR precession. The preces-

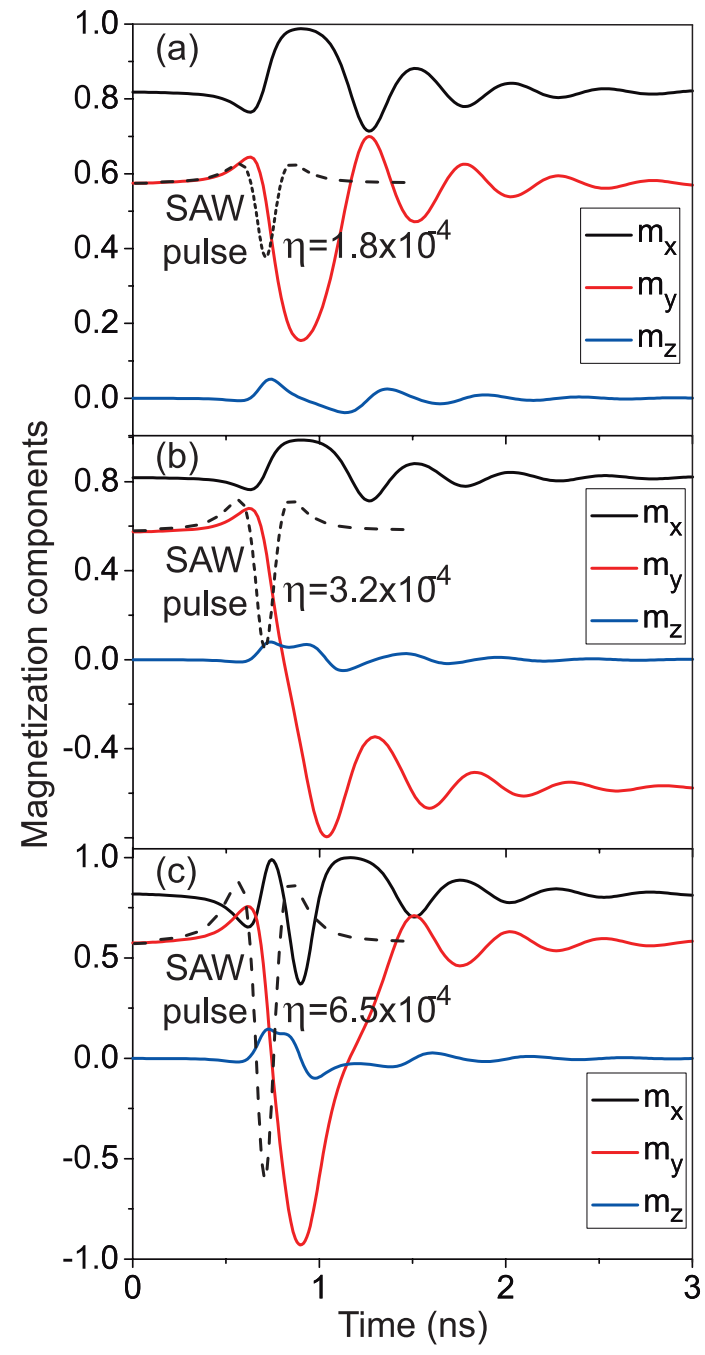

FIG. 3. Magnetization dynamics and switching induced by a single SAW pulse (dashed line) with different amplitudes: (a) no switching at $\eta=1.8 \times 10^{-4}$, (b) switching at $\eta=3.2 \times 10^{-4}$, (c) no switching at $\eta=6.5 \times 10^{-4}$. Duration of the SAW pulse is $\tau=280 \mathrm{ps}$, the pulse is centered at $t_{0}=0.8 \mathrm{~ns}$. Magnetic field is $B=30 \mathrm{mT}$.

sion period increases in the vicinity of the critical field, as dictated by Fig. 2(b), reaching 1 ns at $B=35 \mathrm{mT}$. Figure 4(b) demonstrates a remarkable rapid decrease of $m_{y}$ from +0.7 to -0.9 within approximately $250 \mathrm{ps,}$ i.e. on a deeply sub-cycle time scale. Meanwhile, with regard to the most intriguing question, i.e. on the ultimate speed of magnetization dynamics and switching, here we would like to provide a qualitative explanation based on Fig. 2(b). On the time scale exceeding the acoustic travel time through the nanomagnet (equal to $\left.b / v_{S A W}=100 \mathrm{~nm} / 5.4 \mathrm{~nm} / \mathrm{ps} \simeq 18.5 \mathrm{ps} \ll \tau=280 \mathrm{ps}\right)$, the action of an ultrashort acoustic strain pulse results in an alternating tension and compression of the nanomagnet. It is evident from Fig. 2(c) that the transient compression results in the disappearance of two metastable energy states in a single state, i.e. a transient and reversible phase transition will take place. While in a single min- 


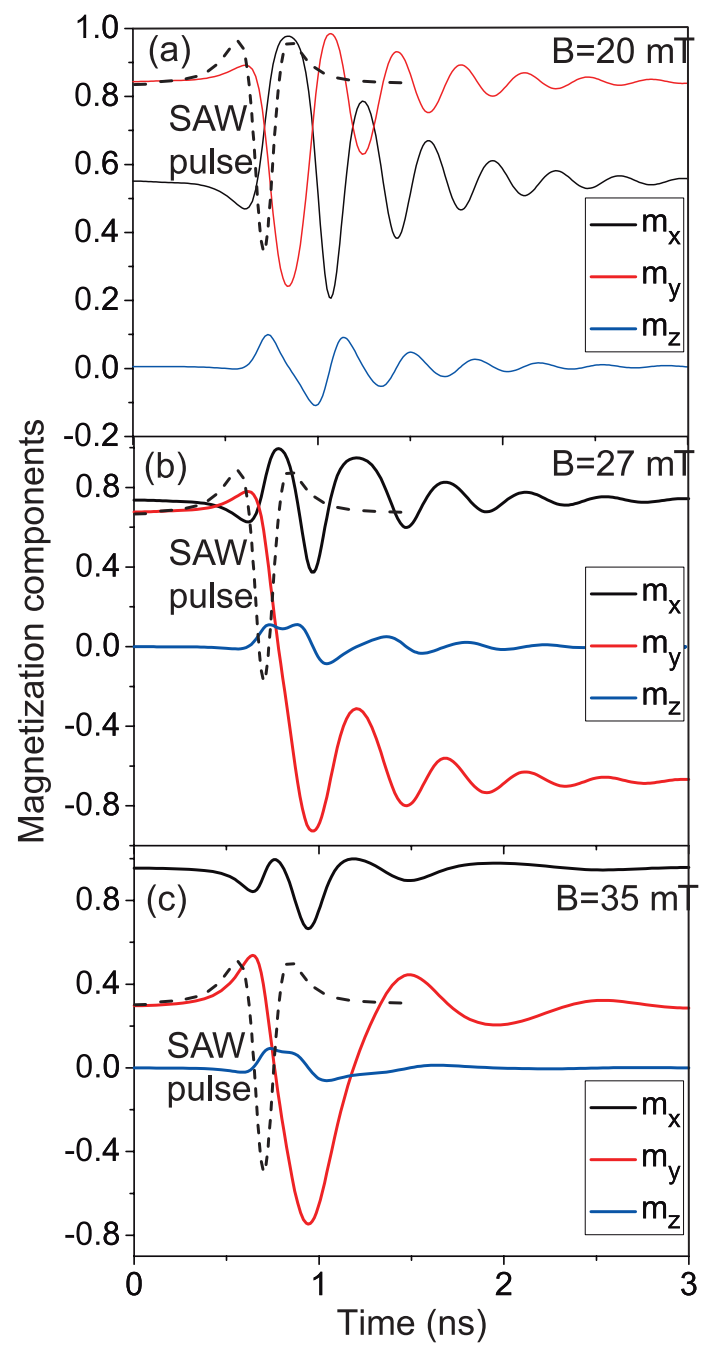

FIG. 4. Magnetization dynamics and switching induced by a single SAW pulse (dashed line) with constant amplitude $\eta=5 \times 10^{-4}$ for three different values of magnetic field: (a) no switching at $B=20 \mathrm{mT}$, (b) switching at $B=27 \mathrm{mT}$, (c) no switching at $B=35 \mathrm{mT}$. Duration of the SAW pulse is $\tau=280 \mathrm{ps}$, the pulse is centered at $t_{0}=0.8 \mathrm{~ns}$.

imum state, the magnetization follows the precessional motion around the $x$-axis. That is why the relevant time scale would be governed by the precessional motion in this strongly perturbed (stressed) single-minimum state and not by the small-angular precession frequency shown in Fig. 2(b).

More insight in the switching behaviour can be inferred from the magneto-elastic switching diagrams displaying the final magnetization state after the interaction with an acoustic pulse. Previously reported magnetoelastic switching diagrams $[22,28]$ represent binary images in a sub-space of physical parameters, indicating solely whether switching occurs or not. Here we introduce a different type of magneto-elastic switching-time diagrams, which also show the characteristic time scale of magnetization switching. Assuming that initially the magnetization rests in the metastable state with $m_{y}>0$
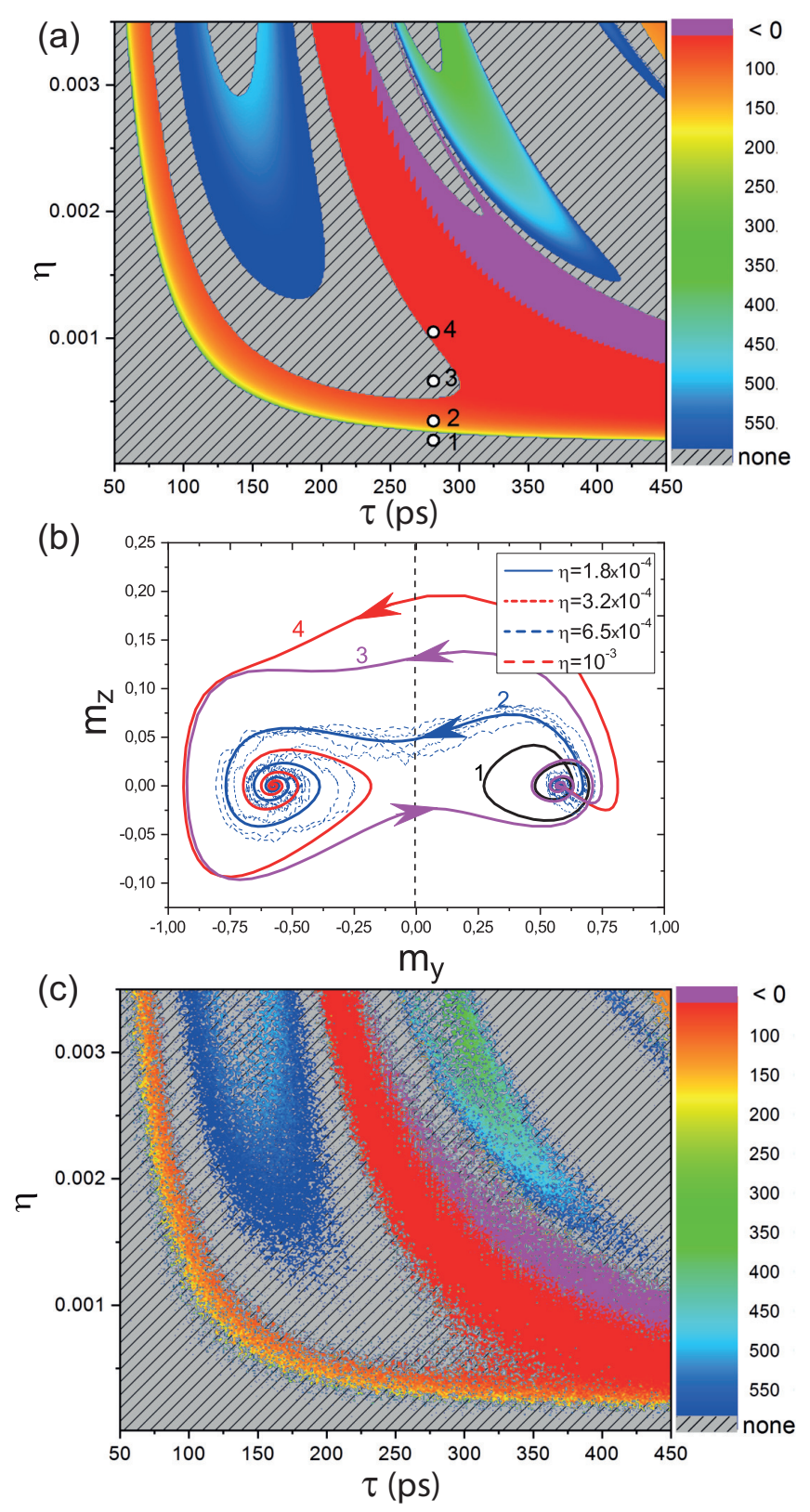

FIG. 5. (a) Magneto-elastic $\eta-\tau$ switching-time diagram for $B=30 \mathrm{mT}$. Dashed areas are not switched, the color within the switched areas maps the switching time in picoseconds (see the color bar and text for details). (b) Magnetization trajectories for different strain amplitudes $\eta=1.8,3.2,6.5,10 \times 10^{-4}$. The switching time for $\eta=3.2 \times 10^{-4}$ and $\eta=10 \times 10^{-4}$ is $260 \mathrm{ps}$ and $170 \mathrm{ps}$, respectively. The duration of the SAW pulse $\tau=280 \mathrm{ps}$. Dashed lines show five switching trajectories under the influence of random thermal noise (see text for details). Panel (c) demonstrates how thermal noise affects the magneto-elastic $\eta-\tau$ switching-time diagram: the boundaries between different regions look fuzzy, while the structure of the diagram persists.

(see Fig. 5(b) and 6(b)), we define the switching time as the delay between the central minimum of the SAW pulse and the sign change of the magnetization component $m_{y}$, if later it does not become positive again. At 

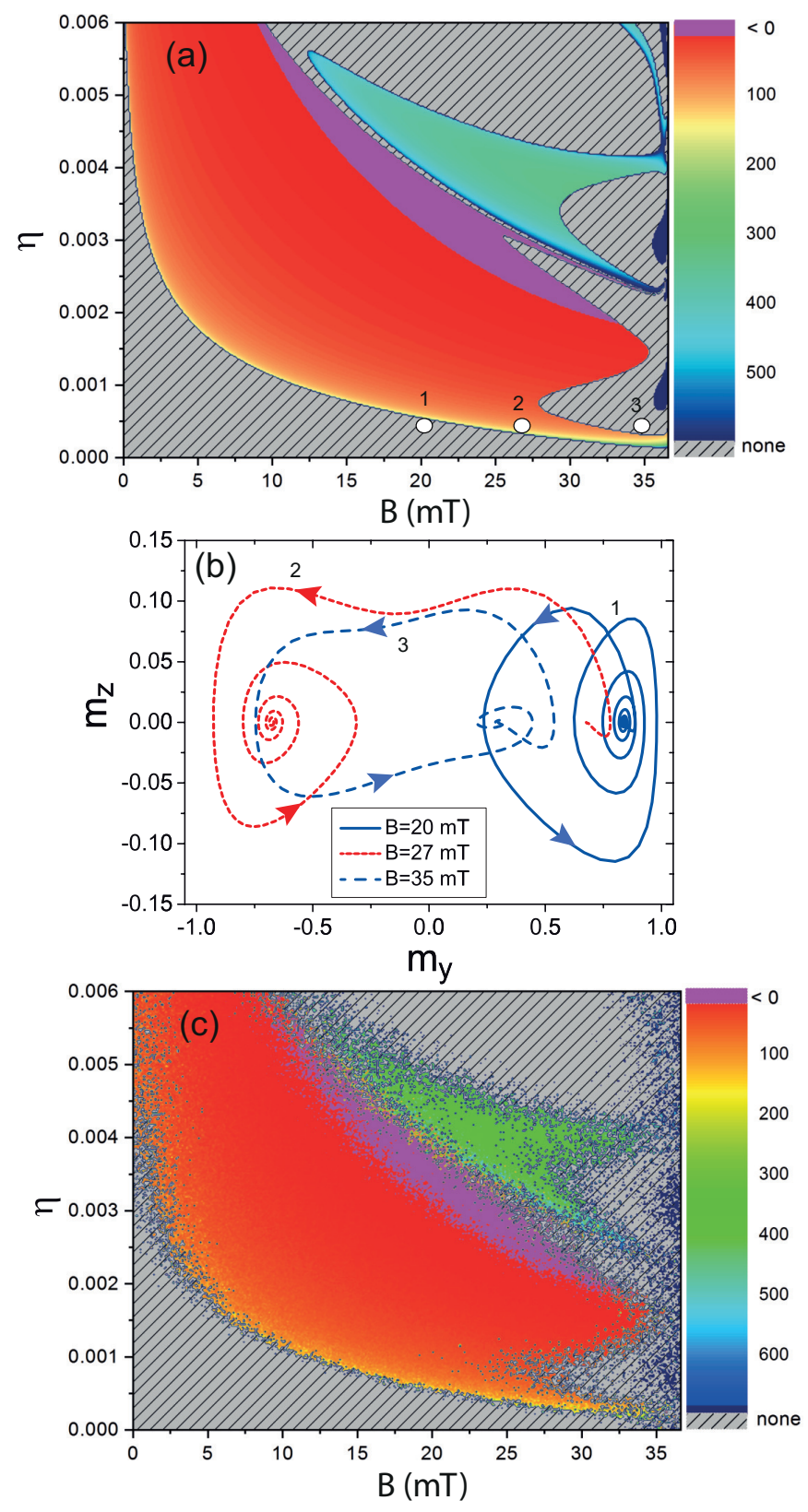

FIG. 6. (a) Magneto-elastic $\eta-B$ switching-time diagram for $\tau=280$ ps. Dashed areas are not switched, the color maps within the switched areas maps the switching time in picoseconds (see text for details). (b) Magnetization trajectories for different values of the magnetic field $B=20,27,35 \mathrm{mT}$ and the SAW amplitude $\eta=5 \times 10^{-4}$. Panel (c) demonstrates how thermal noise affects the magneto-elastic $\eta-B$ switching-time diagram: the boundaries between different regions are particularly affected for smaller $(B<5 \mathrm{mT})$ and larger $(B>33 \mathrm{mT})$ magnetic fields.

first glance, it seems that such definition should result in the underestimation of the switching time, because the damped precession around the second metastable state with $m_{y}<0$ can take hundreds of picoseconds. However, these dynamics can be suppressed with a weaker timedelayed second acoustic pulse via the coherent control of the FMR precession, a well-understood phenomenon in ultrafast magneto-acoustics [39]. Moreover, this definition of switching allows for negative switching times meaning that $m_{y}$ changes the sign even before the strain peak has arrived, i.e. switching occurs clearly within the SAW pulse width.

Fig. 5(a) demonstrates a diagram with the dependence of the switching time on the amplitude $\eta$ and duration $\tau$ of the acoustic pulse. Figure 5(b) displays some representative magnetization trajectories corresponding to $\tau=280 \mathrm{ps}$ and different $\eta$.

The switching time does not vary continuously with the parameters $\eta$ and $\tau$. One can distinguish different areas in Fig. 5(a), appearing in different colors corresponding to switching times covering a wide range between negative (violet), sub-100 ps (red) to above-500 ps (blue). These areas are separated by dashed regions, where the magnetization does not switch. In the faster (violet and red) areas the switching occurs within a fraction of a precession period. In the slower areas it makes one or more rotations around the initial equilibrium state before turning to its final state. Negative and sub- 100 ps switching times in Fig. 5 are significantly shorter than the stationary precession period of 500 ps extracted from Eq. (15) for $B=30 \mathrm{mT}$ and shorter than the acoustic pulse duration $\tau=280 \mathrm{ps}$. This observation highlights the highly non-equilibrium nature of the switching process, where at every time instant the magnetization vector is precessing with time-dependent frequency around the rapidly varying effective magnetic field.

The height of the potential barrier of $11.3 k T$ for $B=30 \mathrm{mT}$ is not so large in comparison with the energy of thermal fluctuations. In order to study the influence of thermal fluctuations on the magneto-elastic switching we have complemented the LLG equations with $\delta(t)$ correlated Langevin forces $\delta H(t)$ randomly changing in 2 ps time steps [40-42]. Thin dashed lines in Fig. 5(b) represent five different realizations of magnetization dynamics in the presence of Langevin noise: all trajectories starting from point 2 in Fig. 5(a) display switching. Figure 5(c) represents a switching diagram calculated in the presence of Langevin noise: each pixel corresponds to a single trajectory of the magnetization vector. Whereas the borders between different zones become fuzzy, the general structure of the switching diagram is preserved proving the robustness of the proposed concept against thermal fluctuations.

The magneto-elastic switching diagram represented in the $\eta-B$ plane (Fig. 6) displays much larger zones indicating that switching should occur for a wide range of parameters, notably for small values of the magnetic field $B<B_{c r} \simeq 37 \mathrm{mT}$. The magnetization trajectories all start from metastable states determined by the magnitude of the magnetic field, see the time traces for Fig. 6(b). The structure of the switching diagram in Fig. 6(a) becomes much more complex for magnetic fields approaching the critical field $B_{c r}$ when height of the barrier between two metastable states gradually disappears and the corresponding switching times exceed $1 \mathrm{~ns}$. 
The switching diagram under in the presence of thermal noise (Fig. 6(c)) shows that the zones of the switching diagram disappear for $B>33 \mathrm{mT}$ where the potential barrier becomes comparable with $k T$ and thermal fluctuations can induce the stochastic switching process. Surprisingly, thermal noise also affects the switching diagram at small fields below $5 \mathrm{mT}$, where the potential barrier is much larger (see Fig. 2). The explanation roots in the fact that the magneto-elastic driving field $\propto \epsilon_{x x} m_{x}$ is proportional to $m_{x}$ that turns zero at $B=0$ and the initial magnetization dynamics out of the equilibrium direction (parallel to the $y$-axis) is driven solely by thermal noise. A better physical understanding might be expected from the analysis of the presented model system within the framework of the dynamic and thermodynamic phase transitions [43].

The magneto-elastic switching should be also sensitive to the geometrical imperfections of lithographically fabricated nanomagnets. Variations in the dimensions of an elliptical nanomagnet will affect the demagnetization tensor and, as a consequence, change the critical field $B_{c r}$ and the height of the potential barrier. For instance, an increase of a large axis of the ellipse from 150 to $160 \mathrm{~nm}$ lifts up the critical field $B_{c r}$ from 37 to $41.5 \mathrm{mT}$ and rises the height of the potential barrier from 70 to $109 \mathrm{kT}$ at $B=20 \mathrm{mT}$. However, the decrease of the magnetic field to $16 \mathrm{mT}$ brings the potential barrier back to $70 \mathrm{kT}$ and restores the conditions for magneto-elastic switching. Figure 6 displays relatively large switched areas within a wide range of magnetic fields. Therefore we conclude that the same acoustic pulse should switch magnetization in nanomagnets with slightly different dimensions. However, as the width of the switched areas in Fig. 6 varies as a function of acoustic pulse duration and amplitude we can only provide a rough estimate that lithographically feasible $10 \%$ tolerances in the lateral dimensions $a$ and $b$ should preserve the switching phenomenon.

The efficiency of the magneto-elastic coupling $\propto b_{1} / M_{0}$ in Eq. (7) is determined by the ratio of the magnetoelastic constant $b_{1}$ and the saturation magnetization $M_{0}$ [22]. The $\sim 10^{-4}$ SAW-induced switching threshold for elliptical Ni nanomagnets appears to be significantly lower as compared to highly magnetostrictive thin films of Terfenol-D switchable by ultrashort longitudinal acoustic pulses with amplitudes $\sim 10^{-2}$ propagating in the direction perpendicular to the surface [22]. This observation may seem surprising because of the $\sim 20$ times larger magnetostriction coefficient in Terfenol-D [44]. However, the much longer $\sim 300 \mathrm{ps}$ duration of the SAW pulses as compared to Ref. [22] and possibility to tune the height of the potential barrier with the external magnetic field overcompensate the lower efficiency of the magneto-elastic coupling in nickel. It is quite straightforward that the SAW-induced switching threshold of Terfenol-D elliptical nanomagnets of similar dimensions should drop below $\sim 10^{-5}$ making the investigated configuration suitable for low-power acoustic transducers.

In this investigations we have restricted ourselves only to analysis of acoustically induced FMR precession
$[1,4,39,45,46]$, i.e. neglecting possible contributions of acoustically excited exchange magnons. We have recently shown that exchange magnons can be efficiently excited only in the case when the spatial extent of acoustic pulses in propagation direction is smaller that the characteristic size of magnetic nanostructure in the propagation direction [47]. In the present investigation acoustic pulses are longer than $100 \mathrm{~nm}$ size suggesting that magneto-elastic excitation of exchange magnons can indeed be neglected.

\section{CONCLUSIONS}

We investigated the phenomenon of magnetization reversal (switching) in elliptical nanomagnets induced by ultrashort SAW pulses of surface acoustic waves. The switching threshold between two metastable singledomain magnetization states depends on the amplitude and duration of SAW pulses, the magneto-elastic coupling efficiency and the height of the potential barrier between these states. The latter is determined by the magnetic shape anisotropy of an elliptical nanomagnet, which depends on its dimensions and the amplitude of the external magnetic field. The key point is tuning the height of the potential barrier between the metastable energy minima using weak magnetic fields easily accessible in the experiments. Our geometry, where the magnetic nanoparticle is driven by short SAW pulses, is complementary to similar objects driven by quasi-monochromatic excitations and displaying bifurcations and chaos [27]. Ultimate speed limits of the magneto-acoustic switching can be investigated solving the LLG equations (7-12) driven by dynamic SAW strains in Eq. (18) and analysing the results within the framework of switching-time diagrams, with or without thermal noise.

The most critical assumption of the present study, i.e. the single-domain magnetic ground state of elliptic nanomagnets, depends on its dimensions and exchange stiffness $[48,49]$ as well as the presence of nano-scale material defects [44]. Therefore, the experimental realization of the proposed SAW-induced magnetic recording will ultimately involve investigations of elliptical nanomagnets with different aspect ratios and dimensions.

\section{ACKNOWLEDGMENTS}

Stimulating discussions with R. Tobey and D. Seletskiy are greatfully acknowledged. The work was financially supported by Strategie internationale "NNNTelecom" and the Acoustic HUB de la Region Pays de La Loire, ANR-DFG "PPMI-NANO" (ANR-15-CE24-0032 and DFG SE2443/2), RFBR (17-57-150001, 17-02-01138, 19-02-00682), Act 211 Government of the Russian Federation (contract 02.A03.21.0011), PRC CNRS-RFBR "Acousto-magneto-plasmonics", Deutsche Forschungsgemeinschaft (AL2143/2-1). 
[1] A. V. Scherbakov, A. S. Salasyuk, A. V. Akimov, X. Liu, M. Bombeck, C. Brüggemann, D. R. Yakovlev, V. F. Sapega, J. K. Furdyna, and M. Bayer, Phys. Rev. Lett. 105, 117204 (2010).

[2] J.-W. Kim, M. Vomir, and J.-Y. Bigot, Phys. Rev. Lett. 109, 166601 (2012).

[3] J. Jäger, A. Scherbakov, T. Linnik, D. Yakovlev, M. Wang, P. Wadley, V. Holy, S. Cavill, A. Akimov, A. Rushforth, and M. Bayer, Appl. Phys. Lett 103, 032409 (2013).

[4] M. Bombeck, A. S. Salasyuk, B. A. Glavin, A. V. Scherbakov, C. Brüggemann, D. R. Yakovlev, V. F. Sapega, X. Liu, J. K. Furdyna, A. V. Akimov, and M. Bayer, Phys. Rev. B 85, 195324 (2012).

[5] T. L. Linnik, A. V. Scherbakov, D. R. Yakovlev, X. Liu, J. K. Furdyna, and M. Bayer, Phys. Rev. B 84, 214432 (2011).

[6] M. Deb, E. Popova, M. Hehn, N. Keller, S. Mangin, and G. Malinowski, Phys. Rev. B 98, 174407 (2018).

[7] L. Thevenard, I. S. Camara, J.-Y. Prieur, P. Rovillain, A. Lemaitre, C. Gourdon, and J.-Y. Duquesne, Phys. Rev. B 93, 140405(R) (2016).

[8] J. Janušonis, C. Chang, P. van Loosdrecht, and R. Tobey, Appl. Phys. Lett. 106, 181601 (2015).

[9] J. Janušonis, T. Jansma, C. L. Chang, L. Q., A. Gatilova, A. M. Lomonosov, V. Shalagatskyi, T. Pezeril, V. V. Temnov, and R. I. Tobey, Scientific Reports 6, 29143 (2016).

[10] J. Janušonis, C. L. Chang, T. Jansma, A. Gatilova, V. S. Vlasov, A. M. Lomonosov, V. V. Temnov, and R. I. Tobey, Phys. Rev. B 94, 024415 (2016).

[11] C. L. Chang, A. M. Lomonosov, J. Janusonis, V. S. Vlasov, V. V. Temnov, and R. I. Tobey, Phys. Rev. B 95, 060409(R) (2017).

[12] K. Uchida, T. An, Y. Kajiwara, M. Toda, and E. Saitoh, Appl. Phys. Lett. 99, 212501 (2011).

[13] M. Weiler, H. Huebl, F. S. Goerg, F. D. Czeschka, R. Gross, and S. T. B. Goennenwein, Phys. Rev. Lett. 108, 176601 (2012).

[14] M. Weiler, L. Dreher, C. Heeg, H. Huebl, R. Gross, M. S. Brandt, and S. T. B. Goennenwein, Phys. Rev. Lett. 106, 117601 (2011).

[15] L. Thevenard, C. Gourdon, J. Y. Prieur, H. J. von Bardeleben, S. Vincent, L. Becerra, L. Largeau, and J. Y. Duquesne, Phys. Rev. B 90, 094401 (2014).

[16] L. Dreher, M. Weiler, M. Pernpeintner, H. Huebl, R. Gross, M. S. Brandt, and S. T. B. Goennenwein, Phys. Rev. B 86, 134415 (2012).

[17] S. Davis, J. A. Borchers, B. B. Maranville, and Adenwalla, J. Appl. Phys. 117, 063904 (2015).

[18] V. Sampath, N. D'Souza, D. Bhattacharya, G. M. Atkinson, S. Bandyopadhyay, and J. Atulasimha, Nano Lett. 16, 5681 (2016).

[19] L. Thevenard, I. S. Camara, S. Majrab, M. Bernard, P. Rovillain, A. Lemaître, C. Gourdon, and J.-Y. Duquesne, Phys. Rev. B 93, 134430 (2016).

[20] P. Kuszewski, I. S. Camara, N. Biarrotte, L. Becerra, J. von Bardeleben, W. S. Torres, A. Lemaître, C. Gourdon, J.-Y. Duquesne, and L. Thevenard, J. Phys.: Condens. Matter 30, 244003 (2018).

[21] K. Roy, S. Bandyopadhyay, and J. Atulasimha, Phys. Rev. B 83, 224412 (2011).
[22] O. Kovalenko, T. Pezeril, and V. V. Temnov, Phys. Rev. Lett. 110, 266602 (2013).

[23] V. V. Temnov, C. Klieber, K. A. Nelson, T. Thomay, V. Knittel, A. Leitenstorfer, D. Makarov, M. Albrecht, and R. Bratschitsch, Nature Communications 4, 1468 (2013).

[24] M. Sander, M. Herzog, J. E. Pudell, M. Bargheer, N. Weinkauf, M. Pedersen, G. Newby, J. Sellmann, J. Schwarzkopf, V. Besse, V. V. Temnov, and P. Gaal, Phys. Rev. Lett. 119, 075901 (2017).

[25] M. Sander, J. Pudell, M. Herzog, M. Bargheer, R. Bauer, V. Besse, V. Temnov, and P. Gaal, Appl. Phys. Lett. 111, 261903 (2017).

[26] X. Li, D. Lobanowski, S. Selahuddin, and C. S. Lynch, J. Appl. Phys. 122, 043904 (2017).

[27] A. M. Ferona and R. E. Camley, Phys. Rev. B 95, 104421 (2017).

[28] E. M. Chudnovsky and R. Jaafar, Phys. Rev. Applied 5, 031002 (2016).

[29] R. Landauer, IBM J. Res. Dev. 5, 183 (1961).

[30] J. Hong, B. Lambson, S. Dhuey, et al., IBM J. Res. Dev. 2, e1501492 (2016)

[31] A. K. Biswas, H. Ahmad, J. Atulasimha, and S. Bandyopadhyay, Nano Letters 17, 3478 (2017).

[32] L. L. Yan, T. P. Xiong, K. Rehan, F. Zhou, D. F. Liang, L. Chen, J. Q. Zhang, W. L. Yang, Z. H. Ma, and M. Feng, Phys. Rev. Lett. 120, 210601 (2018).

[33] J. A. Osborn, Phys. Rev. 67, 351 (1945).

[34] B. Heinrich, Spin Relaxation in Magnetic Metallic Layers and Multilayers, Chapter 5 in: Bland J.A.C., Heinrich B. (eds) Ultrathin Magnetic Structures III., pp. 143-210 (2005).

[35] Q. Li, C. W. Kartikowati, S. Horie, T. Ogi, T. Iwaki, and K. Okuyama, Scientific Reports 7, 9894 (2017).

[36] A. G. Every, Meas. Sci. Techn. 13, R21 (2002).

[37] N. Chigarev, T. Dehoux, C. Rossignol, B. Audoin, and V. Levin, Journal of Physics: Conference Series 92, 012029 (2007).

[38] J. Higuet, T. Valier-Brasier, T. Dehoux, and B. Audoin, Rev. Sci. Instr. 82, 114905 (2011).

[39] J.-W. Kim and J.-Y. Bigot, Phys. Rev. B 95, 144422 (2017).

[40] D. V. Berkov, Magnetization dynamics including thermal fluctuations: Basic phenomenology, fast remagnetization processes and transitions over high-energy barriers in: Kronmüller, H., Parkin, S. (eds) Handbook of magnetism and advanced magnetic materials. Vol.2: Micromagnetism, pp. 795-823 (2007).

[41] D. A. Garanin, Phys. Rev. B 98, 144425 (2018).

[42] A. Roe, D. Bhattacharya, and J. Atulasimha, Appl. Phys. Lett. 115, 112405 (2019).

[43] P. Riego, P. Vavassori, and A. Berger, Physica B 549, 13 (2018).

[44] M. A. Abeed, J. Atulasimha, and S. Bandyopadhyay, J. Phys.: Condens. Matter 30, 394001 (2018).

[45] V. N. Kats, T. L. Linnik, A. S. Salasyuk, A. W. Rushforth, M. Wang, P. Wadley, A. V. Akimov, S. A. Cavill, V. Holy, A. M. Kalashnikova, and A. V. Scherbakov, Phys. Rev. B 93, 214422 (2016).

[46] J.-W. Kim, M. Vomir, and J.-Y. Bigot, Scientific Reports 5, 8511 (2015).

[47] V. Besse, A. V. Golov, V. S. Vlasov, A. Alekhin, 
D. Kuzmin, I. V. Bychkov, L. N. Kotov, and V. V. Temnov, J. Magn. Magn. Mater. (2020).

[48] P.-O. Jubert and R. Allenspach, Phys. Rev. B 70, 144402 (2004).
[49] C. Chen, B. D. Fu, M. E. Dannecker, R. U. Curiel, G. P. Carman, and A. E. Sepulveda, Multifunctional Materials 1, 014001 (2018). 